\title{
A Method of Lifting-out FIB prepared TEM-ready Specimen
}

\section{S. X. Wang and D. Li}

Micron Technology Inc., Boise, ID 83707, USA

Focused ion beam (FIB) systems have been used extensively in failure analysis of semiconductor devices and sample preparation for transmission electron microscopy (TEM) in different applications [1-3]. FIB lift-out is one of the commonly used procedures for making site-specific TEM specimens $[1,2,4]$. In this work, we describe a method for direct lifting-out of a TEM-ready sample.

This method requires a fine-tipped lift-out probe $(<1 \mu \mathrm{m}$ in diameter is ideal). This fine tip can be created by adding a small extension to the original probe [5]. A membrane (about $100 \mathrm{~nm}$ thick, depending on the application) is first milled out by FIB. The linkage to the bulk material is cut except for a single corner, as shown in Fig. 1A. The fine tipped probe is then moved slowly to touch the membrane lengthwise (Fig. 1A) so that they have a contact along the length of the probe tip. Static Van der Waals force attracts the membrane to the tip upon contact. At this point, the corner linkage can be cut by the ion beam. Without the linkage or other objects nearby, the membrane stays attached to the probe tip and can be lifted out of the trench (Fig. 1B). A Cu half-grid (cut from a whole grid by a razor blade or dedicated cutter) with carbon film coating is used as a mounting medium in this demonstration. The grid is loaded into the FIB so that it is standing vertically with the open end on top (Fig. 1C). Other materials can also be used as mounting media. The media must have an open edge, preferably one with an edge thickness small enough to be electron-beam transparent in TEM. A dummy TEM sample is also a good mounting medium. The membrane on the probe tip is brought close to the edge of the carbon film (Fig. 1D). In order to make a good contact, it is necessary to find a location on the carbon film with a relatively flat surface. The membrane is brought into contact with the carbon film so that they partially overlap to each other, but the area of interest for TEM work is left outside the overlapping area. When the overlapped area of membrane-carbon is larger than that of membrane-probe, the adhesion force of the membrane toward the carbon film is also greater. Thus, the probe can be retracted, leaving the membrane attached to the carbon film (Fig. 2A).

The sample can now be safely loaded into the TEM. Fig. 2B shows a TEM image of the membrane on the edge of the carbon film. The area of interest can be studied without obstruction from the carbon film (Fig. 2C). Since the membrane is mounted on the open end of the half-grid, it can also be put back into FIB for further thinning. When re-thinning the specimen, take care to constrain the FIB milling to the open part of the membrane- the area which overlaps the carbon film should not be milled by ion beam. Fig. 2D shows the TEM image of the same sample after additional thinning.

\section{References:}

[1] L. A. Giannuzzi, J. L. Drown, S. R. Brown, R. B. Irwin, and F. A. Stevie, Mat. Res. Soc. Symp. Proc. 480 (1997) 19.

[2] L. R. Herlinger, S. Chevacharoenkul, and D. C. Erwin, Proc. 22nd Int. Symp. Test. Fail. Ana. (1996) 199.

[3] R. J. Young, P. D. Carleson, X. Da, T. Hunt, and J. F. Walker, Proc. 24th Int. Symp. Test. Fail. Ana. (1998) 329.

[4] J. C. Lee, D. Su, and J. H. Chuang, Microelec. Reliab. 41 (2001) 1551. 
[5] S. X. Wang, current proceeding.

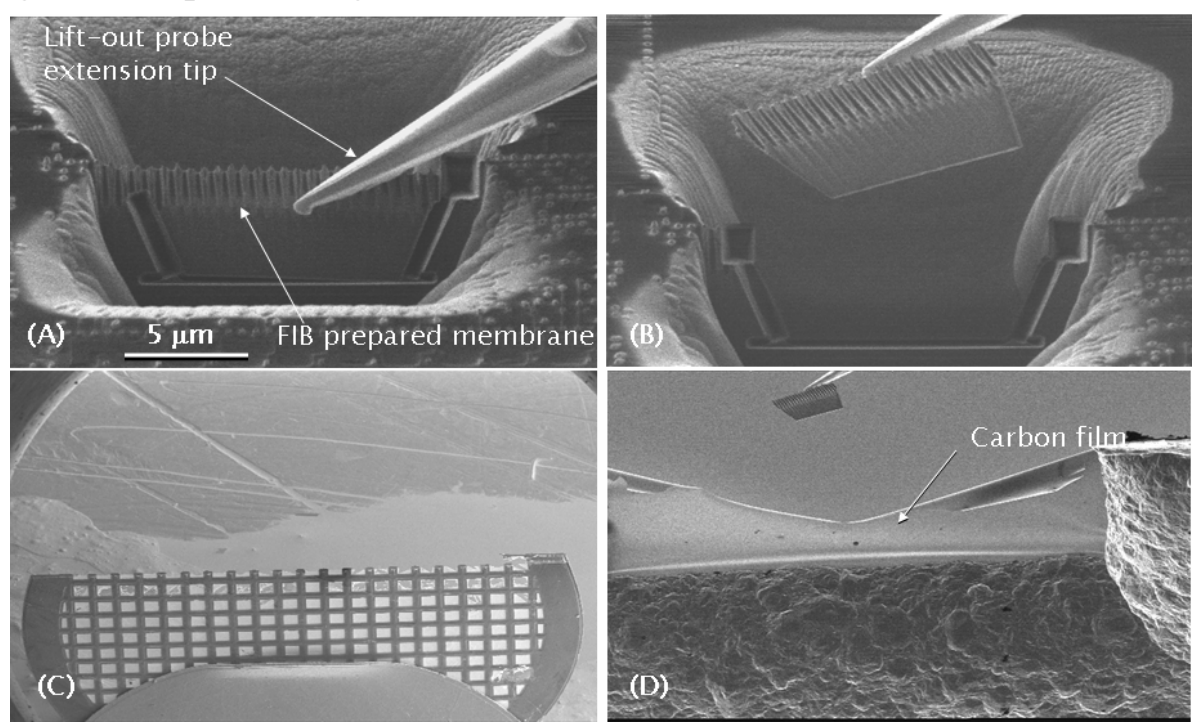

Fig. 1. (A) A FIB-created membrane and the lift-out probe tip. (B) Membrane attaches to the tip by static force and is cut loose. (C) A Cu half-grid with carbon film as the mounting medium. (D) The lifted-out membrane is brought near the edge of the carbon film.

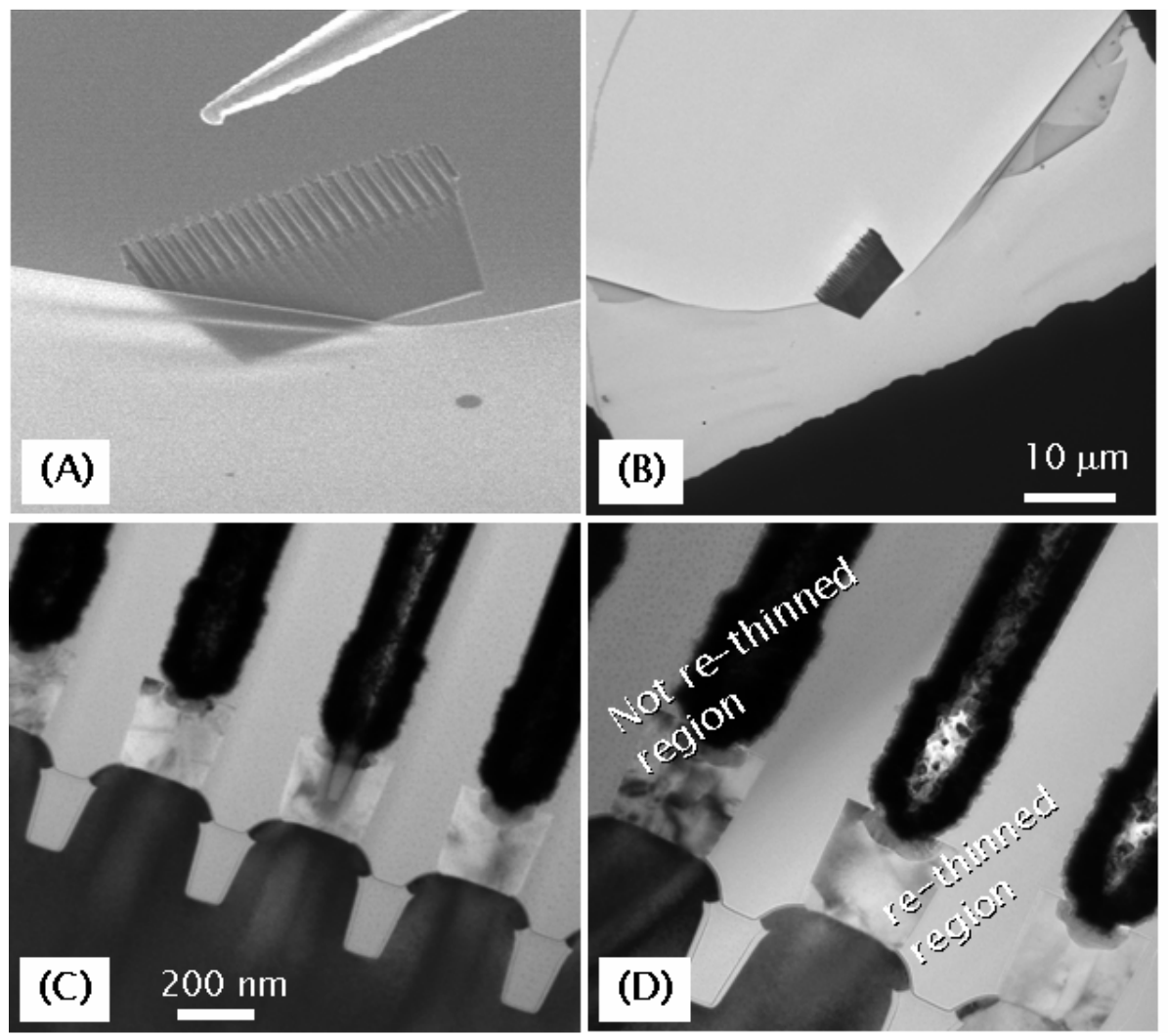

Fig. 2. (A) Membrane overlaps partially with the carbon film. The tip can be retracted without the membrane, due to a stronger adhesion between the membrane and the carbon film. (B) TEM image of the membrane on the edge of carbon film. (C) TEM image of the area unobstructed by carbon film. (D) The membrane-on-carbon film was put into FIB to be re-thinned. The TEM image shows both the original area and the re-thinned area. 